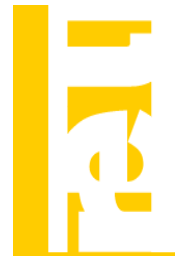

\title{
DETERMINANTES DE LA CONTRATACIÓN INDEFINIDA EN ESPAÑA: NACIONALES VERSUS EXTRANJEROS
}

\author{
Antonio Caparrós *1, Lucía Navarro* \\ *Universidad de Málaga
}

Recibido el 3 de marzo de 2009; aceptado el 17 de mayo de 2010

\section{Resumen}

La reciente evolución de la inmigración en España es un tema de interés dada la magnitud del fenómeno. La aportación económica de los inmigrantes depende del papel que desempeñen en nuestro mercado laboral y, particularmente, de la calidad de sus empleos. En este contexto, se pretende evaluar los factores influyentes sobre la estabilidad laboral, considerando la nacionalidad de los trabajadores. La metodología utilizada consiste en la estimación de modelos logit y en la descomposición de la diferencia de probabilidad media de tener un contrato indefinido entre trabajadores españoles y extranjeros. La información estadistica empleada proviene de la EPA (INE, 2005-2009).

Palabras clave: Inmigración, estabilidad laboral, modelos logit, descomposición de Yun.

Clasificación JEL: J15, J41, J44.

\section{Abstract}

The recent evolution of immigration in Spain and its economic effects are subjects of interest given the magnitude of the phenomenon. The economic contribution and the integration of the immigrants in the Spanish society depend on the quality of their works. In this context, the aim of this paper is to analyse factors affecting employment stability, taking into account the nationality of the workers. The methodology used is the logit model estimation and decomposition of the difference in average probability of having an openended contract between Spanish and foreign workers. Data are obtained from the last waves of EPA (INE, 2005-2009).

Key words: Immigration, employment stability, logit models, Yun decomposition.

JEL Classification: J15, J41, J44.

\footnotetext{
${ }^{1}$ Autor para correspondencia: antonio@uma.es. Este trabajo se ha realizado en el marco de los proyectos: PRY116/09 del Centro de Estudios Andaluces, SEJ2007-68045-CO2-01/ECON del Ministerio de Educación y Ciencia, y P09SEJ4859 de la Junta de Andalucía. Asimismo, los autores agradecen los valiosos comentarios y sugerencias realizados por dos evaluadores anónimos y por el editor de la revista, que sin duda han contribuido a la mejora de este trabajo.
}

(C) Revista de Economía Laboral 


\section{Introducción}

El fuerte ritmo de expansión de la contratación temporal, acaecido desde la reforma laboral de 1984, ha provocado que el fomento de la contratación indefinida sea uno de los principales instrumentos de política activa de empleo implementado en todas las reformas laborales realizadas en España desde 1997. No obstante, este impulso legislativo del empleo estable no ha redundado en una significativa disminución de la tasa de temporalidad, ya que en el año 2009 ésta se sitúa en torno al $25 \%$, lo que contrasta con el 14\% que se alcanza en la UE27.

La creciente evolución del empleo temporal en España ha coincidido con los cambios producidos en los flujos migratorios durante la década de los noventa, que han transformado a nuestro país en una zona de inmigración. Algunos datos significativos que evidencian este hecho son, por un lado, que el peso de la población extranjera sobre la población total ha pasado del 1\% en 1991 hasta el 12\% en el 2009 (INE, 2009). Por otro lado, España junto a Alemania son los países de la UE27 donde los extranjeros tienen una mayor importancia dentro de la población ocupada. Concretamente, en nuestro país el $14,4 \%$ de los empleados han sido extranjeros durante el 2008 (EUROSTAT, 2009). La concurrencia temporal entre el aumento de los flujos migratorios hacia España y el incremento de la precariedad laboral puede representar un obstáculo que ralentice el proceso de asimilación de la población inmigrante y afecte negativamente al desarrollo económico, a la competitividad y al empleo en España.

En este sentido, este artículo pretende analizar si los factores que influyen sobre la contratación indefinida en España muestran una incidencia distinta sobre los trabajadores extranjeros y españoles, ambos grupos con una antigüedad en el empleo inferior a tres años. De esta manera, se pretende arrojar evidencia empírica sobre un aspecto concreto del proceso de asimilación laboral de los inmigrantes escasamente tratado en la literatura económica española. Para el logro de este objetivo, en primer lugar, se estiman modelos de elección discreta teniendo en cuenta las características del trabajador, del empleo, la zona geográfica donde se desempeña la relación laboral y el año en que los individuos son encuestados. En segundo lugar, se procede a la aplicación de una generalización de la descomposición de Oaxaca-Blinder (Blinder, 1973 y Oaxaca, 1973), concretamente, se realiza la descomposición de Yun (Yun, 2004) para modelos no lineales. A partir de esta metodología, se podrá conocer qué parte de la diferencia existente en la probabilidad media de acceder a un contrato indefinido entre españoles y extranjeros se debe a 
las distintas características de cada grupo y qué proporción es consecuencia de las diferentes repercusiones de esas características. La fuente estadística utilizada corresponde a los $2^{\circ}$ trimestres de la EPA (INE) referidos al periodo 2005-2009².

El resto del trabajo está estructurado de la siguiente forma. En la sección 2 se presenta una revisión de la literatura económica existente sobre la asimilación laboral de los inmigrantes en España. En el siguiente apartado se realiza un análisis descriptivo de la muestra utilizada. En los epígrafes 4 y 5 se exponen, respectivamente, la especificación del modelo econométrico y la descomposición de Yun (2004), analizándose en ambos casos los resultados obtenidos de las estimaciones. En la sección 6 se sintetizan las principales conclusiones del estudio. Por último, se añade un anexo con información descriptiva adicional.

\section{Literatura sobre la asimilación laboral de los inmigrantes en España.}

Los procesos de integración y de asimilación de los inmigrantes en el mercado laboral del país de acogida han sido, tradicionalmente, algunos de los principales tópicos en la literatura económica sobre migración (véanse, por ejemplo, los trabajos seminales realizados por Chiswick, 1978; Borjas, 1994 o Zimmermann, 1995).

En España, el fenómeno migratorio ha sido abordado por la literatura económica desde distintos puntos de vista. En primer lugar, una serie de trabajos analizan, bajo un enfoque teórico, los beneficios y costes de la incorporación de los inmigrantes en el mercado de trabajo y en la economía (algunas referencias significativas son, por ejemplo, Dolado, 2002; Vicéns, 2005 y Cuadrado et al., 2007).

En segundo lugar, estudios como Dolado et al. (1998) o AmuedoDorantes y De la Rica (2008a, 2008b) evalúan, desde un punto de vista empírico, la influencia de la presencia de la población inmigrante sobre la economía y el mercado de trabajo español. Así, por un lado, Dolado et al. $(1998)^{3}$ analizan los efectos de la inmigración sobre la demanda de empleo

2 El inicio del periodo coincide con el año 2005, debido a las variaciones metodológicas acaecidas en dicho año en la EPA. Concretamente, se incorporó la nueva normativa europea proveniente de EUROSTAT, se introdujeron mejoras en el método de recogida de la información y se tuvo en cuenta el aumento del número de extranjeros residentes. Para esto último, se sustituyó la base poblacional de los datos del Censo de 1991 por la del Censo de 2001.

${ }^{3}$ Este trabajo sigue la línea de investigación iniciada por Schmidt et al. (1991) y Bauer y Zimmermann (1995). 
y los salarios de los trabajadores españoles en mercados laborales no competitivos, distinguiendo entre mano de obra cualificada y no cualificada. La información estadística utilizada por dichos autores corresponde a los registros administrativos de permisos de trabajo para trabajadores extranjeros (Ministerio de Trabajo, 1990-1992). La principal evidencia que extraen es la existencia de una asociación negativa entre inmigración y los niveles salariales y de empleo de los trabajadores nativos no cualificados. Por otro lado, Amuedo-Dorantes y De la Rica (2008a) estudian la aportación de los extranjeros a la renta nacional, haciendo uso del Censo de Población (INE, 2001), la EPA (INE, 2002) y la Encuesta de Estructura Salarial (INE, 2002). Sus resultados revelan que la contribución de los inmigrantes al PNB del año 2007 es del 0,04\%, en términos globales, aunque por regiones existen diferencias significativas, siendo las comunidades de Cataluña, Valencia, Madrid y Murcia donde la participación de los extranjeros al PNB es mayor, con cifras que oscilan entre el $0,04 \%$ y el 0,25\%. Finalmente, Amuedo-Dorantes y De la Rica (2008b) verifican para la economía española la hipótesis propuesta por Peri y Sparber (2008), consistente en que un incremento de la proporción de extranjeros provoca una reasignación de los trabajadores nativos hacia ocupaciones con un bajo contenido en tareas manuales. En este caso, la fuente estadística utilizada es la EPA (INE 1999-2007).

Un tercer grupo de estudios analizan la incorporación y convergencia laboral de los inmigrantes hacia los trabajadores españoles desde diversas perspectivas. Por un lado, cabe citar los trabajos de Fernández y Ortega (2008) y, Amuedo-Dorantes y De la Rica (2007). En el primero de ellos, se verifica la existencia de diferencias entre la población nativa y la extranjera con respecto a la probabilidad de participar en el mercado de trabajo y encontrar un empleo con datos de la EPA (INE, 1996-2005). Sus resultados muestran que los trabajadores inmigrantes tienen niveles de participación laboral y desempleo más altos que los nativos, aunque la diferencia con ellos prácticamente se anula a partir de los cinco años. En el segundo estudio, con datos del Censo de Población (INE, 2001) y de la Encuesta de Estructura Salarial (INE, 2002), se examina si la asimilación ocupacional de los inmigrantes es una función creciente con su tiempo de permanencia en España. Sus resultados más relevantes son que los inmigrantes masculinos y femeninos, con menos de un año de residencia, experimentan una probabilidad de estar ocupados inferior en 14 y 7 puntos porcentuales, respectivamente, a la de los nativos. Estos diferenciales prácticamente desaparecen en un periodo de dos años para los varones y de uno para las mujeres. Además, también obtienen que los trabajadores españoles realizan tareas mejor remuneradas que los inmigrantes, incluso cinco años después de la llegada 
de éstos a España, siendo las diferencias salariales más amplias entre el colectivo de mujeres. Por otro lado, trabajos como Simón et al. (2008) y Navarro y Rueda (2008) abordan la presencia de diferencias salariales entre nativos e inmigrantes, utilizando datos procedentes de la Encuesta de Estructura Salarial (INE, 2002) y de la Muestra Continua de Vidas Laborales (MTAS, 2006), respectivamente. En ambos estudios se obtiene que las variaciones salariales entre trabajadores españoles y extranjeros son explicadas en gran medida por las diferencias existentes entre sus características observadas.

En este contexto, el trabajo que aquí se realiza aborda en profundidad, desde un punto de vista microeconométrico, un aspecto de la asimilación laboral de los inmigrantes escasamente tratado en la literatura económica existente en España. En particular, se contrasta si los efectos de los determinantes del empleo indefinido en contraposición con el temporal difieren entre la población autóctona y la inmigrante. De esta forma se tendrá una aproximación del grado de segmentación laboral que presentan ambos colectivos; es decir, se verificará si la presencia de la población inmigrante es relativamente mayor en un mercado de trabajo secundario caracterizado por empleos de poca calidad y escasas oportunidades de promoción. Esta asociación entre temporalidad y precariedad laboral es una predicción de la teoría de los mercados segmentados (Doeringer y Piore 1971), que en el caso de España ha sido contrastada, por ejemplo, en trabajos como Amuedo-Dorantes (2000) y De la Rica (2004).

\section{Datos}

La muestra seleccionada está compuesta por trabajadores asalariados de la EPA correspondientes a los $2^{\circ}$ trimestres del periodo 2005-20094. A partir de dicha muestra inicial, sólo se escogen a los trabajadores con menos de tres años de antigüedad, lo cual es habitual en la literatura sobre temporalidad en España (véase, por ejemplo, De la Rica 2004; Davia y Hernanz, 2004 y Montellón, 2008), de esta manera se

\footnotetext{
${ }^{4}$ Como se trabaja con la EPA trimestral, los datos que se extraen para realizar las estimaciones acaban formando un "pooled data". En este caso, las rotaciones de individuos que se producen en la encuesta impiden tratarla directamente como un panel. Lo habitual en los trabajos empíricos es seleccionar sólo las observaciones correspondientes a los segundos trimestres, descartando las del resto de trimestres. Así, se minimiza el problema de repetición de individuos. No obstante, hay que tener en cuenta que la repetición de un individuo en la muestra no implica la repetición de las observaciones, pues las características individuales y laborales pueden variar en el tiempo.
} 
garantiza que los trabajadores ocupen puestos de trabajo en circunstancias económicas, coyunturales y con marcos de relaciones laborales similares. Posteriormente, con objeto de homogeneizar en la mayor medida posible a los colectivos de trabajadores nacionales y extranjeros que se comparan, se realiza un filtro, de forma similar a Fernández y Ortega (2008), que excluye a los inmigrantes que llegaron a España antes de los 16 años (edad legal para trabajar) y a los individuos con doble nacionalidad.

En el cuadro 1 se proporciona el análisis descriptivo de las características personales de los individuos, distinguiéndolos según su nacionalidad: españoles, nacionales de la UE15 y de EEUU, del resto de países de la UE, los procedentes de países europeos no integrados en la $\mathrm{UE}$, africanos y sudamericanos ${ }^{5}$.

La distribución de los individuos según su género muestra que los varones alcanzan su mayor peso dentro los trabajadores africanos, con un $79 \%$, lo que contrasta con el $45 \%$ que se registra en el colectivo de sudamericanos. En relación al nivel educativo, cabe destacar que la mayor acumulación de capital humano se presenta en el colectivo de trabajadores de la UE15 y de EEUU. Así, por ejemplo, el 24\% de los mismos tienen estudios universitarios de ciclo largo. Por el contrario, los individuos que carecen de estudios o sólo tienen estudios primarios se concentran principalmente en los grupos de sudamericanos y africanos, donde representan el 20\% y el 53\%, respectivamente. Finalmente, para las variables que recogen el estado civil y el ser cabeza de familia, se observa en todas las comunidades de extranjeros que los individuos con pareja y los que son cabeza de familia muestran una participación mayor sobre el total de individuos, en relación a la correspondiente para los españoles.

\footnotetext{
5 Los trabajadores procedentes de Asia han sido excluidos del análisis, ya que sólo representan el 0,2\% de los individuos que componen la muestra resultante tras aplicar los filtros anteriores.
} 


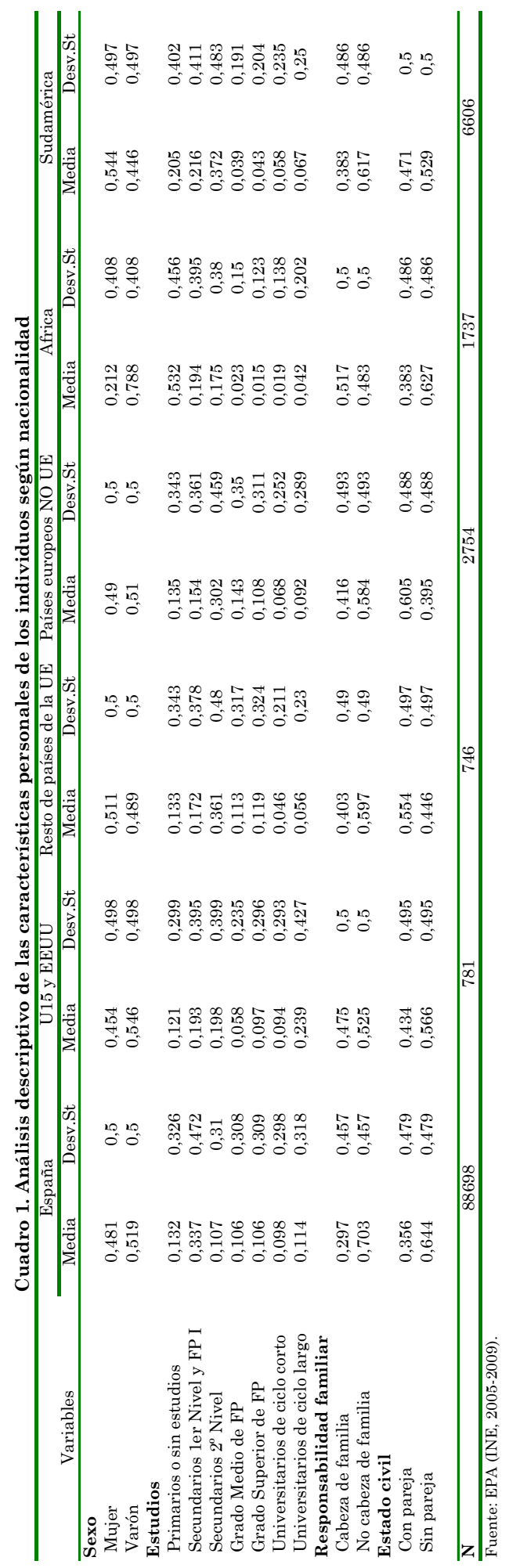


En la tabla A1 del apéndice se informa sobre el análisis descriptivo de las variables explicativas utilizadas para estimar la probabilidad de tener un contrato indefinido frente a uno temporal. Además de la nacionalidad $y$ el resto de características personales, citadas anteriormente, aparecen las características laborales (edad potencial de actividad laboral $^{6}$, antigüedad, ocupación, actividad del establecimiento, tipo de jornada ${ }^{7}$ ), la comunidad autónoma de residencia de los individuos y variables ficticias temporales que recogen el año en que el trabajador es encuestado.

La distribución del total de trabajadores por tipo de contrato refleja una tasa de temporalidad del $60 \%$. Los colectivos nacionales cuya tasa de temporalidad se sitúa por debajo de la anterior cifra son los españoles y los trabajadores de la UE15 y de EEUU, registrando valores del 59\% y del $53 \%$, respectivamente. El resto de grupos presentan tasas superiores al $60 \%$, correspondiendo el mayor porcentaje de temporalidad a los trabajadores africanos con un $75 \%$.

\section{Probabilidad de tener un contrato indefinido frente a uno temporal}

La especificación econométrica utilizada para cuantificar la influencia que ejercen las variables explicativas señaladas anteriormente sobre la probabilidad de tener una relación laboral indefinida es la correspondiente a un modelo logit:

$$
P\left(Y_{i}=1\right)=\frac{\exp \left(\beta^{\prime} X_{i}\right)}{1+\exp \left(\beta^{\prime} X_{i}\right)}
$$

donde $\mathrm{Y}_{\mathrm{i}}$ es igual a 1 si el trabajador es indefinido, y 0 si es temporal; $\mathrm{X}_{\mathrm{i}}$ es un vector que recoge los valores de las variables explicativas para cada individuo y $\beta$ es el vector de parámetros desconocidos que hay que estimar.

En el cuadro 2 aparecen los coeficientes estimados de este modelo, así como sus efectos marginales para el total de asalariados con menos de 3 años de antigüedad en la empresa (primer grupo de columnas) y para la

\footnotetext{
6 La edad potencial de actividad es el tiempo de residencia en España en el caso de los extranjeros, y el tiempo en el mercado de trabajo en el caso de los españoles. Este último se calcula como la diferencia entre la edad actual y la edad a la que terminaron los estudios (Garrido y Toharia, 2003).

${ }^{7}$ La variable tipo de jornada (completa o parcial) hace referencia al contrato principal del trabajador.
} 
segmentación entre españoles y los grupos de extranjeros cuyos coeficientes han resultado significativos; es decir, trabajadores de países europeos distintos a la UE15, Africa y Sudamérica. Los contrastes estadísticos efectuados muestran la idoneidad del modelo, ya que la mayoría de los coeficientes y efectos marginales son significativos. Los resultados sobre las variables de nacionalidad para el total de la muestra indican que los colectivos de inmigrantes citados anteriormente tienen menos opciones de tener un contrato indefinido, sobre todo los trabajadores africanos que tienen una probabilidad de tener un contrato indefinido inferior en 15 puntos porcentuales a los españoles. $\mathrm{Al}$ parecer los empresarios son más reacios a la estabilización de los trabajadores inmigrantes pertenecientes a economías menos desarrolladas que la española, quizás porque puedan tener un riesgo de retorno al país de origen ${ }^{8}$ o una posible mayor movilidad laboral voluntaria. Estos motivos contrarrestarían el efecto de la mayor motivación inicial que puedan tener los trabajadores extranjeros con respecto a los nativos.

A fin de observar ahora si existen diferencias cuantitativas y/o cualitativas en la influencia del resto de variables sobre el tipo de contrato, en función de la procedencia del individuo, nos centramos en los dos últimos grupos de columnas del cuadro $2^{9}$. En relación al sexo, los resultados difieren según la nacionalidad de los sujetos. Así, los varones españoles tienen 3,7 puntos más de probabilidad de ser indefinidos que las mujeres, mientras que si son inmigrantes presentan 3,5 puntos menos de opciones de serlos. Este diferente comportamiento puede explicarse, por un lado, porque las mujeres inmigrantes presentan menos interrupciones en sus trayectorias laborales ${ }^{10} \mathrm{y}$, por otro lado, porque muchas de ellas se dedican al servicio doméstico, donde hay una mayor propensión a las relaciones laborales estables.

\footnotetext{
8 Este riesgo proviene de que el trabajador extranjero puede considerar que su decisión de inmigrar ha sido equivocada o que la haya realizado por un periodo corto de tiempo, por ejemplo para adquirir cualificaciones.

${ }_{9}$ Los descriptivos correspondientes a las variables explicativas para ambas muestras se incluyen en las tablas A2 y A3 del anexo.

10 Un dato estadístico que puede corroborar esta hipótesis es que la tasa de actividad de las mujeres españolas en el año 2009 es del 49\%, mientras que la de las mujeres inmigrantes es del $69 \%$.
} 
Cuadro 2. Estimación logit de la probabilidad de tener un contrato indefinido

\begin{tabular}{|c|c|c|c|c|c|c|}
\hline \multirow[t]{2}{*}{ Variables } & \multicolumn{2}{|c|}{ Total asalariados (a) } & \multicolumn{2}{|c|}{ Asalariados españoles } & \multicolumn{2}{|c|}{$\begin{array}{l}\text { Asalariados de países europeos } \\
\text { no UE15, Africa y Sudamérica }\end{array}$} \\
\hline & Coef. & Ef.Marg & Coef. & Ef.Marg & Coef. & Ef.Marg \\
\hline \multicolumn{7}{|l|}{ Nacionalidad } \\
\hline UE15 o EEUU & 0,124 & 0,029 & & & & \\
\hline Resto de países de UE & $-0,393^{* * *}$ & $-0,087^{* * *}$ & & & & \\
\hline Resto de Europa & $-0,379^{* * *}$ & $-0,084^{* * *}$ & & & & \\
\hline Africa & $-0,713^{* * *}$ & $-0,149^{* * *}$ & & & & \\
\hline Sudamérica & $-0,092^{* *}$ & $-0,021^{* *}$ & & & & \\
\hline \multicolumn{7}{|l|}{ Sexo } \\
\hline Varón & $0,114^{\star * *}$ & $0,026^{* * *}$ & $0,158^{* * *}$ & $0,037^{* * *}$ & $-0,163^{* *}$ & $-0,035^{* * *}$ \\
\hline \multicolumn{7}{|l|}{ Estudios } \\
\hline Secundarios 1er Nivel y FPI & $0,120^{* * * *}$ & $0,028^{* * * *}$ & $0,120^{* * *}$ & $0,029^{* * *}$ & $0,198^{* *}$ & $0,044^{* *}$ \\
\hline Secundarios $2^{\circ}$ Nivel & $0,216^{* * *}$ & $0,051^{* * *}$ & $0,241^{* * *}$ & $0,059^{* * *}$ & $0,255^{* * *}$ & $0,056^{* * *}$ \\
\hline Grado Medio de FP & $0,261^{* * *}$ & $0,062^{* * *}$ & $0,292^{* * *}$ & $0,070^{* * *}$ & 0,131 & 0,029 \\
\hline Grado Superior de FP & $0,305^{* * *}$ & $0,073^{* * *}$ & $0,333^{* * *}$ & $0,082^{\star * * *}$ & $0,277^{\text {**** }}$ & $0,063^{* *}$ \\
\hline Universitarios de ciclo corto & $0,310^{* * *}$ & $0,074^{* * * *}$ & $0,362^{* * *}$ & $0,088^{* * * *}$ & 0,093 & 0,02 \\
\hline Universitarios de ciclo largo & $0,348^{\star * *}$ & $0,084^{* * *}$ & $0,376^{* * *}$ & $0,091^{* * *}$ & $0,395^{* * *}$ & $0,091^{* * *}$ \\
\hline \multicolumn{7}{|l|}{ Estado civil } \\
\hline Con pareja & $0,286^{* * *}$ & $0,067^{* * *}$ & $0,304^{* * *}$ & $0,072^{* * *}$ & $0,106^{* *}$ & $0,023^{* *}$ \\
\hline Cabeza de familia & $0,166^{* * *}$ & $0,039^{* * *}$ & $0,192^{* * *}$ & $0,046^{* * *}$ & 0,016 & 0,003 \\
\hline \multicolumn{7}{|l|}{ Edad potencial de actividad } \\
\hline Entre 1 y 3 años & $0,126^{* * *}$ & $0,030^{* * *}$ & $0,187^{* * *}$ & $0,044^{* * *}$ & $-0,05$ & $-0,01$ \\
\hline Más de 3 años & $0,355^{* * * *}$ & $0,081^{* * *}$ & $0,444^{* * *}$ & $0,101^{* * *}$ & 0,103 & 0,022 \\
\hline Antigüedad en el empleo (meses) & $0,088^{* * * *}$ & $0,020^{* * *}$ & $0,088^{* * *}$ & $0,020^{* * *}$ & $0,085^{* * * *}$ & $0,018^{* * * *}$ \\
\hline \multicolumn{7}{|l|}{ Ocupación } \\
\hline Directivos de empresas y de las administraciones públicas & $1,252^{* * *}$ & $0,302^{* * *}$ & $1,227^{* * *}$ & $0,296^{* * *}$ & $2,211^{\star * * *}$ & $0,489^{* * *}$ \\
\hline Técnicos y profesionales científicos e intelectuales & $0,161^{1 * * *}$ & $0,038^{* * *}$ & $0,196^{* * *}$ & $0,047^{* * *}$ & $0,029^{* *}$ & $0,006^{* *}$ \\
\hline Técnicos y profesionales de apoyo & $0,457^{* * *}$ & $0,110^{* * *}$ & $0,489^{* * *}$ & $0,119^{* * *}$ & $0,712^{* * * *}$ & $0,169^{* * *}$ \\
\hline Empleados de tipo administrativo & $0,303^{* * *}$ & $0,073^{* * *}$ & $0,335^{* * *}$ & $0,081^{* * *}$ & $0,579^{* * *}$ & $0,136^{* * * *}$ \\
\hline Trabajadores de servicios y vendedores de comercio & $0,389^{* * *}$ & $0,093^{* * *}$ & $0,469^{* * *}$ & $0,113^{* * *}$ & $0,171^{* *}$ & $0,038^{* *}$ \\
\hline Trabajadores cualificados en la agricultura y pesca & $0,482^{* * *}$ & $0,118^{* * * *}$ & $0,455^{* * *}$ & $0,111^{* * *}$ & $0,594^{* *}$ & $0,140^{* *}$ \\
\hline Trabajadores cualificados en la industria & 0,019 & $0,004^{* * *}$ & $0,029^{* * * *}$ & $0,007^{* *}$ & $0,241^{* *}$ & $0,054^{* *}$ \\
\hline Operadores de instalaciones y maquinaria, y montadores & $0,108^{* * *}$ & $0,025^{* * *}$ & $0,126^{* * *}$ & $0,030^{* * *}$ & $0,219^{* *}$ & $0,049^{* *}$ \\
\hline \multicolumn{7}{|l|}{ Actividad del establecimiento } \\
\hline Agricultura, ganadería, pesca, caza... & $-0,634^{* * *}$ & $-0,135^{* * *}$ & $-0,614^{* * *}$ & $-0,132^{* * *}$ & $-0,492^{* * *}$ & $-0,099^{* * *}$ \\
\hline Construcción & $-0,637^{* * *}$ & $-0,139^{* * *}$ & $-0,605^{* * *}$ & $-0,134^{* * *}$ & $-0,718^{* * *}$ & $-0,145^{* * *}$ \\
\hline Comercio y hostelería & $0,287^{* * *}$ & $0,069^{* * *}$ & $0,278^{* * *}$ & $0,066^{* * *}$ & $0,396^{* * *}$ & $0,089^{* * *}$ \\
\hline Transporte & $0,190^{* * *}$ & $0,046^{* * * *}$ & $0,183^{* * *}$ & $0,044^{* * *}$ & 0,21 & 0,047 \\
\hline Intermediación financiera y actividades inmobiliarias & $0,272^{* * *}$ & $0,065^{* * *}$ & $0,267 * * *$ & $0,064^{* * *}$ & $0,337^{* *}$ & $0,077^{* *}$ \\
\hline Administraciones públicas, educación y actividades sanitarias & $-0,719^{* * *}$ & $-0,155^{* * *}$ & $-0,759^{* * *}$ & $-0,165^{* * * *}$ & $-0,015$ & $-0,003$ \\
\hline Otras actividades de servicios & $0,369^{* * * *}$ & $0,089^{* * *}$ & $0,176^{* * *}$ & $0,042^{* * *}$ & $0,960^{* * *}$ & $0,224^{* * * *}$ \\
\hline \multicolumn{7}{|l|}{ Tipo de jornada } \\
\hline Jornada completa & $0,492^{* * *}$ & $0,110^{* * * *}$ & $0,461 * * *$ & $0,104^{* * *}$ & $0,581^{* * * *}$ & $0,118^{* * *}$ \\
\hline Comunidades Autónomas & & & & & & \\
\hline Galicia & $0,305^{* * *}$ & $0,073^{* * *}$ & $0,296 * * *$ & $0,071^{* * *}$ & $0,661^{* * * *}$ & $0,157^{* * *}$ \\
\hline Castilla-La Mancha & $0,689^{* * * *}$ & $0,169^{* * *}$ & $0,700^{* * *}$ & $0,172^{* * *}$ & $0,653^{* * *}$ & $0,154^{* * *}$ \\
\hline Extremadura & $0,255^{* * *}$ & $0,061^{* * *}$ & $0,245^{* * *}$ & $0,059^{* * *}$ & $0,626^{* *}$ & $0,148^{* *}$ \\
\hline Valencia & $0,634^{* * *}$ & $0,155^{* * *}$ & $0,649^{* * * *}$ & $0,159^{* * *}$ & $0,666^{* * *}$ & $0,155^{\text {***}}$ \\
\hline Murcia & $0,469^{* * *}$ & $0,114^{* * *}$ & $0,524^{* * *}$ & $0,128^{* * *}$ & $0,310^{* *}$ & $0,070^{* *}$ \\
\hline Canarias & $0,361^{* * * *}$ & $0,087^{* * *}$ & $0,359^{* * *}$ & $0,087^{* * *}$ & $0,571^{* * *}$ & $0,134^{* * * *}$ \\
\hline Asturias & $0,478^{* * *}$ & $0,117^{* * *}$ & $0,445^{* * *}$ & $0,108^{* * *}$ & $1,014^{* * *}$ & $0,245^{* * * *}$ \\
\hline Cantabria & $0,443^{* * *}$ & $0,108^{* * *}$ & $0,452^{* * *}$ & $0,110^{* * *}$ & $0,434^{* *}$ & $0,101^{* *}$ \\
\hline País Vasco & $0,374^{* * *}$ & $0,090^{* * *}$ & $0,364^{* * *}$ & $0,088^{* * *}$ & $0,471^{* * *}$ & $0,110^{* * *}$ \\
\hline Navarra & $0,621^{* * * *}$ & $0,152^{* * *}$ & $0,572^{* * *}$ & $0,140^{* * *}$ & $1,038^{* * *}$ & $0,250^{* * * *}$ \\
\hline Aragón & $0,781^{* * *}$ & $0,191^{* * *}$ & $0,793^{* * *}$ & $0,195^{* * *}$ & $0,725^{* * *}$ & $0,172^{* * *}$ \\
\hline La Rioja & $0,923^{* * *}$ & $0,226^{* * *}$ & $0,922^{* * *}$ & $0,226^{* * *}$ & $0,987^{* * *}$ & $0,238^{* * * *}$ \\
\hline Madrid & $0,976^{* * *}$ & $0,239^{* * *}$ & $1,020^{* * * *}$ & $0,249^{* * *}$ & $0,871^{* * * *}$ & $0,206^{* * *}$ \\
\hline Castilla-León & $0,718^{* * * *}$ & $0,175^{\text {****}}$ & $0,698^{* * *}$ & $0,171^{* * *}$ & $0,993^{* * *}$ & $0,238^{* * * *}$ \\
\hline Baleares & $0,720^{* * * *}$ & $0,177^{* * *}$ & $0,679 * * *$ & $0,167^{\star * * *}$ & $1,000^{* * *}$ & $0,241^{* * * *}$ \\
\hline Cataluña & $1,013^{* * *}$ & $0,247^{* * *}$ & $1,021^{* * *}$ & $0,249^{* * *}$ & $1,046^{* * *}$ & $0,248^{* * * *}$ \\
\hline Año de observación & & & & & & \\
\hline Año 2006 & $-0,03$ & $-0,007$ & $-0,032$ & $-0,007$ & $-0,012$ & 0,004 \\
\hline Año 2007 & $0,149^{* * *}$ & $0,035^{* * *}$ & $0,132^{* * *}$ & $0,031^{* * *}$ & $0,309^{* * *}$ & $0,075^{* * *}$ \\
\hline Año 2008 & $0,227^{\star * *}$ & $0,054^{* * *}$ & $0,204^{* * *}$ & $0,048^{* * *}$ & $0,408^{* * *}$ & $0,104^{* * *}$ \\
\hline Año 2009 & $0,258^{* * *}$ & $0,062^{* * *}$ & $0,223^{* * *}$ & $0,053^{* * *}$ & $0,493^{* * *}$ & $0,124^{* * *}$ \\
\hline Constante & $-3,473^{* * * *}$ & & $-3,584^{* * *}$ & & $-3,6981^{* *}$ & \\
\hline Test de la Razón de Verosimilitudes & & ***** & & & & \\
\hline $\mathrm{N}^{\circ}$ Observaciones & & & & & & \\
\hline
\end{tabular}


Con respecto al nivel educativo, se verifican las predicciones de la teoría del capital humano sobre la relación positiva entre la acumulación de conocimientos, productividad en el trabajo y éxito en la trayectoria profesional (Becker, 1964; Black y Lynch, 1996). Para los trabajadores españoles, esto ocurre especialmente para los universitarios (tanto de ciclo corto como largo), que presentan una probabilidad de tener un contrato indefinido que es en torno a 9 puntos porcentuales superior a la de los individuos con estudios primarios o sin estudios (categoría de referencia). En el caso de los trabajadores inmigrantes, los individuos con mayores opciones de tener una relación laboral estable son aquellos con estudios de FP de grado superior o universitarios de ciclo largo con probabilidades que son, respectivamente, 6 y 9 puntos superiores a la correspondiente para la categoría de referencia ${ }^{11}$.

En relación a las variables familiares, el hecho de tener pareja o ser cabeza de familia afecta positivamente a la probabilidad de tener un contrato indefinido, si el trabajador es español. La mayor responsabilidad del individuo asociada a ambos estados puede implicar un mayor esfuerzo en el puesto de trabajo por parte del trabajador que contribuya a su mejora profesional. En el caso de los inmigrantes, dichas relaciones sólo se observan para aquellos con pareja, donde dicha situación les hace aumentar 2 puntos porcentuales su probabilidad de conseguir la estabilidad laboral respecto a los individuos sin pareja (en torno a 5 puntos inferior a lo observado para los nacionales).

En cuanto a las variables que recogen las características laborales cabe señalar, en primer lugar, que la edad potencial de actividad laboral ejerce una influencia positiva sobre la probabilidad de tener una relación estable si los trabajadores son españoles. Concretamente, se observa que los individuos con menos de 1 año de experiencia potencial tienen más opciones de tener contratos temporales que el resto. Este resultado es coherente con la teoría que postula que determinados contratos temporales son escalones a superar al inicio de la vida laboral de los individuos (véase, por ejemplo, Booth et al. 2005). Por el contrario para los trabajadores extranjeros, donde esta variable coincide con sus años de residencia en España, los resultados no son significativos, lo que refleja la escasa influencia del aumento del tiempo de permanencia en España sobre

\footnotetext{
${ }^{11}$ Los resultados relativos al nivel de estudios de los inmigrantes hay que considerarlos como una proxy de la verdadera importancia de la educación sobre la probabilidad de tener un contrato indefinido para la población extranjera. Este hecho se debe a la posible aparición de sesgos cuando los inmigrantes equiparan sus niveles educativos adquiridos en sus países de origen con los títulos reconocidos oficialmente en España.
} 
la asimilación laboral de los inmigrantes en términos de estabilidad, sin duda por la gran movilidad laboral externa que han debido tener ${ }^{12}$. Sin embargo, su antigüedad en el empleo sí tiene un efecto positivo sobre su estabilidad y, además, similar que para los nacionales. Concretamente, un incremento de un año en el periodo de permanencia en la empresa está asociado con un aumento de la probabilidad de tener un contrato indefinido en torno a 2 puntos porcentuales. Son varios los factores que justifican este resultado. Por un lado, como indica la teoría del job matching (Jovanovic, 1979), la antigüedad del trabajador en la empresa es una señal de la calidad de su emparejamiento con el empleador. En efecto, conforme mayor sea la duración de la relación laboral cabe esperar que mejor sea ésta y que el individuo haya superado con éxito el periodo de prueba en la empresa, por consiguiente, mayores serán las opciones de que se transforme su relación en estable. Por otro lado, más antigüedad en el puesto de trabajo implicará que el trabajador manifiesta una baja propensión al cambio de empleo voluntario y, por tanto, una mayor fidelidad a la empresa que podría ser premiada con la transformación de su contrato en indefinido.

En segundo lugar, respecto a la relación entre el tipo de ocupación y la modalidad contractual, se observa que los trabajadores no cualificados son los que tienen menos opciones de poseer un empleo indefinido, y esto tanto para los nacionales como para los extranjeros. Por su parte, la actividad del establecimiento, que es un reflejo de la estructura productiva de la economía, señala a la agricultura y a la construcción como los sectores más propensos a la temporalidad. Por último, son los trabajadores a tiempo parcial los que presentan una situación más precaria, que se intensifica para los extranjeros.

La ubicación geográfica del trabajador ejerce una influencia significativa sobre su tipo de contrato. Concretamente, todas las comunidades autónomas tienen una menor propensión al empleo temporal que Andalucía. En el caso de los trabajadores españoles son Cataluña y Madrid las que muestran una mayor divergencia con la comunidad andaluza (en torno a 24 puntos porcentuales). Para el caso de los

\footnotetext{
${ }^{12}$ La robustez de los efectos de las variables de edad potencial de la actividad sobre la probabilidad de tener un contrato indefinido se ha comprobado sustituyendo dichas variables por otras que muestran la edad de los individuos. Los resultados nuevamente indican, por un lado, que la edad tiene un efecto positivo para los trabajadores españoles, mientras que para los trabajadores inmigrantes no es relevante. Por otro lado, la significación y cuantía de los coeficientes estimados del resto de variables explicativas no se ven afectadas. Por tanto, se mantiene como regresor a la edad potencial de la actividad ya que consideramos que aproxima mejor a la experiencia potencial de los individuos en el mercado laboral.
} 
inmigrantes, las mayores diferencias respecto a Andalucía se constatan para las comunidades de Baleares, Cataluña y Navarra donde los trabajadores presentan una probabilidad de tener un contrato indefinido superior en 24 puntos porcentuales a la existente en la comunidad andaluza.

Finalmente, las variables ficticias que recogen el año cuando el individuo es encuestado muestran que, desde el año 2007, los trabajadores tanto españoles como inmigrantes tienen más probabilidad de lograr un empleo estable con respecto a los años 2006 y 2005 . Este resultado pone de manifiesto los efectos positivos que tuvieron las medidas legislativas para el fomento de la contratación indefinida que se implementaron en la última reforma laboral realizada en mayo del 2006 (Real Decreto-Ley 5/2006).

\section{Descomposición de la diferencia de probabilidad media de ser indefinido}

Una vez detectada la existencia de diferencias en el acceso al empleo indefinido entre trabajadores nacionales y extranjeros, a través de la estimación del modelo logit anterior, se procede a la aplicación de la metodología propuesta por Yun (2004) para detectar en qué medida dichas discrepancias se deben a las diferencias entre las dotaciones de características de los trabajadores españoles y extranjeros, o a las distintas repercusiones de dichas características sobre la propensión al empleo indefinido entre ambos colectivos. El método de descomposición propuesto por Yun es una generalización del método de Oaxaca-Blinder (Blinder, 1973 y Oaxaca, 1973) para funciones no lineales, como es el caso de las correspondientes a los modelos logit especificados anteriormente.

Para realizar dicha descomposición, en primer lugar, se utilizan las estimaciones expuestas en el cuadro 2 para obtener las probabilidades medias predichas de tener un contrato indefinido, Pr $o b(C I)_{G}$, donde el subíndice $\mathrm{G}$ es igual a E, para el colectivo español, e I, para el colectivo de inmigrantes ${ }^{13}$. De esta forma la diferencia de probabilidad media se puede expresar en términos de los valores medios de la función de distribución acumulada de la logística, $\overline{F\left(X_{G} \hat{\beta}_{G}\right)}$, como:

\footnotetext{
13 A lo largo de este epígrafe cuando hagamos referencia al colectivo de extranjeros hay que tener en cuenta que en dicho grupo sólo están incluidos los trabajadores procedentes de países europeos distintos de la UE15, de Africa y de Sudamérica.
} 


$$
\overline{\operatorname{Prob}(C I)_{E}}-\overline{\operatorname{Prob}(C I)_{I}}=\overline{F\left(X_{E} \hat{\beta_{E}}\right)}-\overline{F\left(X_{I} \hat{\beta}_{I}\right)}
$$

Esta diferencia de probabilidad media es de 4,6 puntos porcentuales a favor de los trabajadores españoles y ha resultado ser estadísticamente significativa tras la aplicación de un contraste de diferencia de proporciones ${ }^{14}$.

El punto de partida de la metodología propuesta por Yun (2004) consiste en descomponer la diferencia expresada en (2) como la suma de dos términos:

$$
\left.\left.\left.\left.\overline{\operatorname{Pr} o b(C I)_{E}}-\overline{\operatorname{Pr} o b(C I)_{I}}=\overline{\left(F \left(X_{E} \hat{\beta_{E}}\right.\right.}\right)-\overline{F\left(X_{I} \hat{\beta_{E}}\right.}\right)\right)+\overline{\left(F\left(X_{I} \hat{\beta}_{E}\right)\right.}-\overline{F\left(X_{I} \hat{\beta_{I}}\right)}\right)
$$

El primer término recoge el efecto atribuible a las distintas características de los dos grupos de trabajadores, españoles y extranjeros (efecto de las características), mientras que el segundo término cuantifica qué parte de la diferencia total es debida a la distinta valoración que realiza el mercado de las características de los dos colectivos analizados para acceder a un empleo estable (efecto de los coeficientes).

El siguiente paso consiste en detallar la contribución de cada una de las variables explicativas a los dos efectos anteriores; es decir, obtener lo que se denomina descomposición detallada. Yun (2004) propone una metodología general para obtener la descomposición detallada de la expresión (3), que no depende de la forma funcional especificada para la variable dependiente; los únicos requisitos son que la variable dependiente sea una función de una combinación lineal de las variables independientes y que dicha función sea diferenciable, restricciones que se cumplen para los modelos logit especificados. Dicha descomposición detallada es la siguiente:

$$
\left.\left.\left.\left.\overline{\operatorname{Prob}(C I)_{E}}-\overline{\operatorname{Prob}(C I)_{I}}=\sum_{i=1}^{k} W_{\Delta X}^{i} \overline{\left(F \left(X_{E} \hat{\beta}_{E}\right.\right.}\right) \overline{F\left(X_{I} \hat{\beta_{E}}\right)}\right)+\sum_{i=1}^{k} W_{\Delta \beta}^{i} \overline{\left(F \left(X_{I} \hat{\beta_{E}}\right.\right.}\right) \overline{F\left(X_{I} \hat{\beta_{I}}\right)}\right)
$$

\footnotetext{
${ }^{14}$ Las características del mercado laboral español implican que esta diferencia de 4,6 puntos porcentuales no es nada despreciable, dada la alta persistencia de la tasa de temporalidad en España, valga como ejemplo que las reformas laborales de 1997 y 2001 apenas afectaron a dicha tasa, que se situó por encima del 30\% hasta el año 2007.
} 
donde los $\operatorname{pesos}^{15}$ de cada variable $i$ sobre las diferencias en características y en coeficientes son $W_{\Delta X}^{i}=\left(\bar{X}_{E}^{i}-\bar{X}_{I}^{i}\right) \hat{\beta}_{E}^{i} /\left(\bar{X}_{E}-\bar{X}_{I}\right) \hat{\beta}_{E}$ y $W_{\Delta \beta}^{i}=\bar{X}_{I}^{i}\left(\hat{\beta}_{E}^{i}-\hat{\beta}_{I}^{i}\right) / \bar{X}_{I}\left(\hat{\beta}_{E}-\hat{\beta}_{I}\right)$, respectivamente.

Una cuestión que hay que tener en cuenta antes de obtener la descomposición detallada, es que la contribución de las variables ficticias incluidas en el modelo a la diferencia de probabilidad media de tener un contrato indefinido entre españoles y extranjeros no es robusta a la categoría de referencia elegida; es decir, lo que se conoce en la literatura económica como problema de identificación (Oaxaca y Ransom, 1999). Por este motivo, la contribución de cada variable explicativa a la diferencia de probabilidad media se calcula siguiendo el enfoque de Yun (2008), en el cual previamente al cálculo de las distintas ponderaciones se realiza una regresión normalizada, que es el resultado de promediar todas las posibles estimaciones cuando se permutan las categorías de referencia asociadas a cada atributo. De esta forma se obtiene el efecto de cada categoría sobre la probabilidad de tener un contrato indefinido, sujeto a las restricciones de que el conjunto de todos los coeficientes correspondientes a todas las categorías han de sumar cero, que es una restricción similar a la impuesta por Suits (1984) y Gardeazabal y Ugidos (2004) ${ }^{16}$.

Los resultados obtenidos tras realizar la descomposición tanto agregada como detallada (expresiones 2 y 3 , respectivamente) aparecen en el cuadro 3.

El primer hecho significativo que se comprueba es que la contribución de las distintas características entre trabajadores españoles e inmigrantes a la diferencia de probabilidad media de tener un contrato indefinido es muy pequeña, ya que sólo representa el 11\%, siendo dentro de ella el mayor efecto debido a la antigüedad y al efecto global de la ocupación en el empleo (cada una de una de las variables tiene un peso de un $8 \%$ favorable para los españoles). La principal contribución, el $89 \%$ restante, se debe a las diferentes repercusiones

15 Dichos pesos cumplen las siguientes condiciones: $\sum_{i}^{K} W_{\Delta X}^{i}=1$ y $\sum_{i}^{K} W_{\Delta \beta}^{i}=1$.

16 Según Gardeazabal y Ugidos (2004), cuando se comparan dos grupos de individuos, esta restricción implica que los coeficientes que componen las categorías de una variable cualitativa no pueden ser en todas las situaciones superiores en un grupo frente al otro. Esta limitación se atenúa por la posibilidad, ofrecida por la técnica utilizada, de cuantificar el efecto conjunto de la variable cualitativa sobre la discriminación, cálculo que se realiza en este trabajo y que aparece en el cuadro 3 para todas las variables cualitativas. 
que ejercen las características sobre la probabilidad de tener un contrato indefinido; es decir, el efecto debido a las diferencias de coeficientes (componente injustificado). Este resultado conlleva que a continuación nos centremos en los pesos de las distintas repercusiones de las variables más significativas (efecto coeficiente) sobre la diferencia total. Así, en primer lugar, se constata que la experiencia potencial de los individuos ejerce una mayor influencia sobre la obtención de un empleo estable para el colectivo nacional, con una aportación positiva a la diferencia de probabilidad media de un $30 \%$ para los trabajadores con más de 3 años de edad potencial de actividad. Este hecho refleja que la experiencia laboral de los inmigrantes es menos valorada por los empresarios, lo cual puede ser debido a que las barreras idiomáticas y culturales existentes para los trabajadores extranjeros limiten su asimilación de conocimientos válidos para el empleo. En cualquier caso, este resultado muestra que la mera permanencia de los inmigrantes en España no es un elemento que mejore su estabilidad. En segundo lugar, otras características donde su distinta valoración por el mercado presenta una aportación positiva e importante a la diferencia total son el ser varón $(29 \%)$, trabajador de los servicios o vendedor de comercio (34\%), o desempeñar una actividad en la construcción (27\%). Además, cabe destacar que las variables que recogen las comunidades autónomas tienen, de forma conjunta, una aportación positiva de un $34 \%$, siendo los trabajadores españoles residentes en la comunidad de Madrid los más beneficiados por esta característica frente a los trabajadores extranjeros.

En cuanto a las variables cuya influencia sobre la probabilidad del logro de un contrato indefinido es favorable para los inmigrantes, cabe destacar que el ser mujer, no tener pareja y, principalmente, trabajar en el sector denominado otras actividades de servicios presentan una aportación negativa importante. En particular, para ésta última variable su aportación negativa a la diferencia de probabilidad media entre españoles y extranjeros es de un $42 \%$. Este resultado se debe a que el empleo doméstico está incluido en dicho sector, y como ya ha sido corroborado por Martínez (1999) la estabilidad laboral de las trabajadoras extranjeras es muy alta en dicho empleo. Por último, se observa que las variables ficticias temporales registran una aportación negativa a partir del 2007, que se incrementa hasta el 2009 donde se observa una aportación negativa de un 10\%. Esta circunstancia puede ser un indicio de que el impulso a la contratación indefinida que supuso la reforma del 2006 no ha afectado en menor medida a la población inmigrantes que a la española, si no todo lo contrario. 
Cuadro 3. Descomposición de la diferencia de probabilidad media de tener un contrato indefinido entre españoles y extranjeros

\begin{tabular}{|c|c|c|}
\hline Diferencia total (\%) & $\begin{array}{c}\text { Diferencia debida a } \\
\text { características (\%) }\end{array}$ & $\begin{array}{c}\text { Diferencia debida } \\
\text { a coeficientes (\%) }\end{array}$ \\
\hline 100 & 10,87 & 89,13 \\
\hline \multicolumn{3}{|l|}{ Aportación de cada variable a la descomposición total (\%) } \\
\hline Sexo & 0,08 & 1,59 \\
\hline Mujer & 0,04 & $-27,53$ \\
\hline Varón & 0,04 & 29,12 \\
\hline Estudios & 2,07 & $-16,35$ \\
\hline Primarios o sin estudios & 1,88 & $-4,4$ \\
\hline Secundarios 1er Nivel y FPI & $-1,22$ & $-9,06$ \\
\hline Secundarios $2^{\circ}$ Nivel & $-0,21$ & $-7,77$ \\
\hline Grado Medio de FP & 0,2 & 2,47 \\
\hline Grado Superior de FP & 0,39 & 0,05 \\
\hline Universitarios de ciclo corto & 0,47 & 4,1 \\
\hline Universitarios de ciclo largo & 0,56 & $-1,74$ \\
\hline Estado civil & $-4,06$ & 1,68 \\
\hline Sin pareja & $-2,03$ & $-16,63$ \\
\hline Con pareja & $-2,03$ & 18,31 \\
\hline Cabeza de familia & $-1,74$ & $-5,44$ \\
\hline No & $-0,87$ & $-18,18$ \\
\hline Sí & $-0,87$ & 12,74 \\
\hline Edad potencial de actividad & 5,6 & 21,93 \\
\hline Menos de 1 año & 1,93 & $-11,88$ \\
\hline Entre 1 y 3 años & 1,44 & 3,95 \\
\hline Más de 3 años & 2,23 & 29,86 \\
\hline Antigüedad en el empleo (meses) & 7,85 & 12,71 \\
\hline Ocupación & 7,98 & 55,87 \\
\hline Directivos de empresas y de las administraciones públicas & 0,9 & $-0,58$ \\
\hline Técnicos y profesionales científicos e intelectuales & $-0,27$ & 1,5 \\
\hline Técnicos y profesionales de apoyo & 2,03 & $-0,49$ \\
\hline Empleados de tipo administrativo & 0,64 & $-0,9$ \\
\hline Trabajadores de servicios y vendedores de comercio & $-0,25$ & 34,08 \\
\hline Trabajadores cualificados en la agricultura y pesca & $-0,1$ & 0,11 \\
\hline Trabajadores cualificados en la industria & 0,59 & $-3,79$ \\
\hline Operadores de instalaciones y maquinaria, y montadores & $-0,2$ & 1,59 \\
\hline No cualificados & 4,64 & 24,35 \\
\hline Actividad del establecimiento & $-2,99$ & 4,09 \\
\hline Agricultura, ganadería, pesca, caza... & 1,77 & 2,81 \\
\hline Construcción & 2,84 & 26,89 \\
\hline Industria & 0,44 & 8,94 \\
\hline Comercio y hostelería & $-0,22$ & 8,95 \\
\hline Transporte & 0,69 & 2,3 \\
\hline Intermediación financiera y actividades inmobiliarias & 1,88 & 2,56 \\
\hline Administraciones públicas, educación y actividades sanitarias & $-6,98$ & $-5,87$ \\
\hline Otras actividades de servicios & $-3,41$ & $-42,49$ \\
\hline Tipo de jornada & $-0,78$ & $-13,94$ \\
\hline Jornada completa & $-0,39$ & 3,62 \\
\hline Jornada parcial & $-0,39$ & $-17,56$ \\
\hline Comunidades Autónomas & $-1,98$ & 34,41 \\
\hline Andalucía & -4 & 4,74 \\
\hline Galicia & $-0,52$ & $-1,56$ \\
\hline Castilla-La Mancha & 0,07 & 4,54 \\
\hline Extremadura & $-0,51$ & $-0,78$ \\
\hline Valencia & $-0,92$ & 6,14 \\
\hline Murcia & $-0,23$ & 9,41 \\
\hline Canarias & $-0,13$ & $-1,01$ \\
\hline Asturias & $-0,01$ & $-1,37$ \\
\hline Cantabria & 0 & 0,93 \\
\hline País Vasco & $-0,13$ & 0,27 \\
\hline Navarra & $-0,02$ & $-2,67$ \\
\hline Aragón & $-0,37$ & 3,91 \\
\hline La Rioja & $-0,55$ & 0,71 \\
\hline Madrid & 7,44 & 12,21 \\
\hline Castilla-León & 0,31 & $-3,8$ \\
\hline Baleares & $-0,41$ & $-3,18$ \\
\hline Cataluña & -2 & 5,92 \\
\hline Año de observación & $-1,14$ & $-4,68$ \\
\hline Año 2005 & $-0,46$ & 8,47 \\
\hline Año 2006 & $-0,16$ & 7,59 \\
\hline Año 2007 & $-0,03$ & $-3,05$ \\
\hline Año 2008 & $-0,27$ & $-7,54$ \\
\hline Año 2009 & $-0,22$ & $-10,15$ \\
\hline Constante & & $-2,74$ \\
\hline
\end{tabular}

Fuente: Elaboración propia a partir de la EPA (INE 2005-2009). 


\section{Conclusiones}

El estudio realizado ha pretendido arrojar nueva evidencia empírica sobre los procesos de integración y asimilación de la población inmigrante en el mercado de trabajo español. Concretamente, se ha analizado si los factores que influyen sobre la contratación indefinida en España presentan una distinta incidencia en los trabajadores extranjeros y los españoles, haciendo uso de una muestra de asalariados con menos de 3 años de antigüedad procedente de la EPA (INE 20052009). Tras la estimación de un modelo logit, se ha verificado que la incidencia de la contratación indefinida en el colectivo de trabajadores nacionales es superior a la existente para los trabajadores extranjeros procedentes de países europeos distintos a la UE15, Sudamérica y Africa. En particular, los trabajadores africanos tienen una probabilidad de tener un contrato indefinido inferior en 15 puntos porcentuales a la de los españoles. Estos resultados revelan que la nacionalidad es una variable determinante en la posición que ocupa el trabajador dentro del mercado laboral en España.

Posteriormente, se han estimado respectivos modelos logit para los trabajadores españoles y el colectivo de extranjeros compuesto por los grupos citados anteriormente, con objeto de comprobar si los factores que determinan la probabilidad de tener un contrato indefinido difieren entre ambas submuestras. Los resultados obtenidos revelan algunas diferencias significativas. En primer lugar, el sexo ejerce una distinta influencia según la nacionalidad del trabajador; concretamente, los varones españoles tienen 4 puntos más de probabilidad de ser indefinidos que las mujeres, mientras que si son inmigrantes tienen 3,7 puntos menos de probabilidad de serlos. En segundo lugar, la edad potencial de actividad laboral actúa positivamente sobre los asalariados nacionales; por el contrario, los efectos de dicha variable sobre los extranjeros no son significativos. Este último resultado refleja, por un lado, la existencia de barreras para la asimilación de conocimientos útiles para las trayectorias laborales de estos trabajadores. Por otro lado, indica que la permanencia en nuestro mercado de trabajo si no está acompañada de un proceso de adquisición de capital humano específico no afecta a la estabilidad de los trabajadores, puesto que la antigüedad en la empresa (proxy de esta variable) sí ayuda a su estabilidad.

La escasa importancia de la experiencia potencial para los inmigrantes en el acceso al empleo indefinido, se corrobora tras la 
aplicación de la descomposición de Yun (2004) sobre la diferencia existente entre las probabilidades medias de tener un contrato indefinido entre españoles y extranjeros. Concretamente, la distinta repercusión de la variable ficticia que recoge a los trabajadores con una edad potencial de actividad superior a 3 años supone una contribución positiva de un $30 \%$ a la diferencia de probabilidades medias, a favor del colectivo nacional. En este sentido, la instrumentación de políticas de empleo destinadas a facilitar la adquisición de formación fuera de la empresa, por parte de los trabajadores inmigrantes, es uno de los elementos principales para fomentar la asimilación de dichos trabajadores a nuestro mercado laboral. Finalmente, cabe señalar que los resultados relativos a la aportación de las variables ficticias temporales parece revelar que el fomento del empleo estable instrumentado en la reforma del 2006, mediante la ampliación de las bonificaciones a la contratación indefinida, ha contribuido a que las diferencias entre españoles y extranjeros en cuanto al acceso a la estabilidad laboral disminuyan a favor de éstos últimos.

\section{Bibliografía}

Amuedo-Dorantes, C. (2000): "Work transitions into and out of involuntary temporary employment in a segmented market: Evidence from Spain”, Industrial Relations Review, 53 (2), pp. 309-325.

Amuedo-Dorantes, C. and De la Rica, S. (2007): "Labour market assimilation of recent immigrants in Spain", British Journal of Industrial Relations, 45 (2), pp. 257-284.

Amuedo-Dorantes, C. and De la Rica, S. (2008a) : "Does immigration raise natives income? National and regional evidence from Spain”, IZA, Discussion Paper Series n ${ }^{\circ} 3486$.

Amuedo-Dorantes, C. and De la Rica, S. (2008b): "Complements or substitutes? Immigrant and native task specialization in Spain”, Centre for Research and Analysis of Migration, London, UK, Discussion Paper $\mathrm{n}^{\mathrm{o}}$ $16 / 08$.

Bauer, T. and Zimmermann, K. (1995): "Integrating the East: The labour market effects of immigration", CEPR, Working Paper, $\mathrm{n}^{\circ} 1235$.

Becker, G. (1964): Human Capital: A theoretical and empirical analysis, with special references to education, New York: NBER. 
Black, S. and Lynch, L. (1996): "Human capital investment and productivity”, American Economic Review, 86 (2), pp. 263-267.

Blinder, A. S. (1973): "Wage discrimination: reduced form and structural variables", Journal of Human Resources, 8 (4), pp. 436-455.

Booth, A., Francesconi, M., and Frank, J. (2005): "Temporary jobs: Stepping stones or dead ends?" IZA, Discussion Paper Series n ${ }^{\circ} 205$.

Borjas, G. (1994): "The economic benefits from immigration", Journal of Economics Perspective, 9 (2), pp. 3-22.

Chiswick, B. (1978): "The effect of americanization on the earnings of foreing-born men", Journal of Political Economy, 86 (5), pp, 897-921.

Cuadrado, JR., Iglesias, C. y Llorente, R. (2007): Inmigración y mercado de trabajo (1997-2005), Fundación BBVA, Madrid.

Davia, M.A. y Hernanz, V. (2004): “Temporary employment and segmentation in the Spanish Labour market: An empirical analysis through the study of wage differentials", Spanish Economic Review, 6 (4), pp. 291-318.

De la Rica, S. (2004): "Wage gaps between workers with indefinite and fixed-term contracts: The impact of firm and occupational segregation", Moneda y Crédito, 219, pp. 43-68.

Dolado, J. (2002): "Los nuevos fenómenos migratorios: Retos y políticas", capítulo 2 en T. García Milá (ed) Nuevas fronteras de la Política Económica, CREI (UPR) y Generalitat de Cataluña.

Dolado, J., Jimeno, J. y Duce, R. (1998): "Los efectos de la inmigración sobre la demanda relativa de trabajo cualificado versus poco cualificado: Evidencia para España”, Cuadernos Económicos de I.C.E, 63, pp. 11-29.

Doeringer, P. y Piore, M. (1971): Internal labour markets and manpower analysis, Lexington Mass: D. C. Heath.

EUROSTAT (2009): Labour Force Survey.

Fernández, C. y Ortega, C. (2008): "Labor market assimilation of immigrants in Spain: Employment at the expense of bad job-matches? Spanish Economic Review, 10 (2), pp. 83-107.

Gardeazabal, J. y Ugidos, A. (2004): "More on identification in detailed wage decompositions", Review of Economics and Statistics, 86 (4), pp. 1034-1036. 
Garrido, L. y Toharia, L. (2003): "La situación laboral de los españoles y los extranjeros según la Encuesta de Población Activa”, Economistas, 99, pp. 74-86.

INE (2009): Padrón Municipal de Habitantes.

Izquierdo, M., Jimeno, J. y Rojas, J. A. (2007): "On the aggregate effects of immigration in Spain”, Banco de España, Documento de Trabajo n 0714.

Jovanovic, B. (1979): "Job matching and the theory of turnover", Journal of Political Economy, 87 (5), pp. 972-990.

Martínez, U. (1999): "Immigrants in the Spanish labour market", en Baldwin-Edwards, M. y otros (eds) Immigrants and the Informal economy in Southern Europe, London, PORTLAND.

Montellón, E. (2008): "Un análisis de las diferencias regionales en el impacto de la contratación temporal en España”, Investigaciones Regionales, 12, pp. 107-131.

Navarro, Ma.L. y Rueda, M. F. (2008): “¿Sufren discriminación salarial los inmigrantes en España? Una perspectiva regional”, CENTRA, Documento de Trabajo E2008/05.

Oaxaca, R. (1973): "Male-female wage differentials in urban labour markets", International Economic Review, 14 (3), pp. 693-709.

Oaxaca, R. and Ransom, M. (1999): "Identification in detailed wage decompositions", Review of Economics and Statistics, 81 (1), pp. 154-157.

Peri, G. and Sparber, C. (2008): "Task specialization, immigration and wages", CReAM, Discussion Paper $n^{\circ}$ 02/08.

Schmidt, C., Stilz, A. y Zimmermann, K. (1991): "Mass migration, unions and government intervention", Journal of Public Economics, 55 (2), pp. 185-201.

Simón, H., Sanromá, E. y Ramos, R. (2008): "Labour segregation and immigrant native-born wage distributions in Spain: an analysis using matched employer-employee data". Spanish Economic Review, vol. n ${ }^{\circ}$, pp. 135-168.

Suits, D. (1984): "Dummy variables: Mechanics v. interpretation", Review of Economics and Statistics, 66 (1), pp. 177-180.

Vicéns, J. (2005): "Impacto económico de la inmigración sobre el mercado laboral. Una Revisión”, Instituto L.R. Klein-Centro Gauss, U.A.M. D.T. n ${ }^{\circ}$ 10 . 
Yun, M. (2004): "Decomposing differences in the first moment", Economic Letter 82 (2), pp. 275-280.

Yun, M. (2008): "Identification problem and detailed Oaxaca decomposition: A general solution and inference", Journal of Economic and Social Measurement, 33 (1), pp. 27-38.

Zimmermann, K. (1995): "Tackling the European migration problem", Journal of Economic Perspective, 9, pp. 45-62. 


\begin{tabular}{|c|c|c|c|c|}
\hline \multirow{2}{*}{ Variables } & \multicolumn{2}{|c|}{ Temporales } & \multicolumn{2}{|c|}{ Indefinidos } \\
\hline & Media & Desv. Est. & Media & Desv. Est. \\
\hline \multicolumn{5}{|l|}{ Nacionalidad } \\
\hline Españoles & 0,868 & 0,338 & 0,885 & 0,317 \\
\hline UE15 o EEUU & 0,006 & 0,082 & 0,009 & 0,094 \\
\hline Resto de países de UE & 0,008 & 0,085 & 0,007 & 0,085 \\
\hline Resto de Europa & 0,031 & 0,171 & 0,023 & 0,148 \\
\hline Africa & 0,022 & 0,144 & 0,011 & 0,102 \\
\hline Sudamérica & 0,065 & 0,247 & 0,065 & 0,245 \\
\hline \multicolumn{5}{|l|}{ Sexo } \\
\hline Mujer & 0,475 & 0,5 & 0,49 & 0,5 \\
\hline Varón & 0,525 & 0,5 & 0,51 & 0,5 \\
\hline \multicolumn{5}{|l|}{ Estudios } \\
\hline Primarios o sin estudios & 0,25 & 0,423 & 0,117 & 0,302 \\
\hline Secundarios 1er Nivel y FP I & 0,334 & 0,471 & 0,296 & 0,456 \\
\hline Secundarios $2^{\circ}$ Nivel & 0,128 & 0,334 & 0,143 & 0,35 \\
\hline Grado Medio de FP & 0,097 & 0,296 & 0,107 & 0,309 \\
\hline Grado Superior de FP & 0,091 & 0,287 & 0,116 & 0,32 \\
\hline Universitarios de ciclo corto & 0,091 & 0,287 & 0,096 & 0,295 \\
\hline Universitarios de ciclo largo & 0,1 & 0,3 & 0,125 & 0,331 \\
\hline \multicolumn{5}{|l|}{ Responsabilidad familiar } \\
\hline Cabeza de familia & 0,29 & 0,454 & 0,343 & 0,475 \\
\hline No cabeza de familia & 0,71 & 0,454 & 0,657 & 0,475 \\
\hline \multicolumn{5}{|l|}{ Estado civil } \\
\hline Con pareja & 0,353 & 0,478 & 0,411 & 0,492 \\
\hline Sin pareja & 0,647 & 0,478 & 0,589 & 0,492 \\
\hline Edad potencial de actividad & & & & \\
\hline Menos de 1 año & 0,128 & 0,334 & 0,077 & 0,225 \\
\hline Entre 1 y 3 años & 0,141 & 0,348 & 0,117 & 0,322 \\
\hline Más de 3 años & 0,731 & 0,443 & 0,806 & 0,395 \\
\hline Antigüedad en el empleo (meses) & 9,37 & 9,22 & & \\
\hline Ocupación & & & & \\
\hline Directivos de las empresas y de las administraciones públicas & 0,005 & 0,071 & 0,022 & 0,148 \\
\hline Técnicos y profesionales científicos e intelectuales & 0,098 & 0,298 & 0,091 & 0,288 \\
\hline Técnicos y profesionales de apoyo & 0,092 & 0,29 & 0,135 & 0,342 \\
\hline Empleados de tipo administrativo & 0,089 & 0,285 & 0,113 & 0,316 \\
\hline Trabajadores de servicios y vendedores de comercio & 0,188 & 0,39 & 0,221 & 0,415 \\
\hline Trabajadores cualificados en la agricultura y pesca & 0,011 & 0,106 & 0,009 & 0,094 \\
\hline Trabajadores cualificados en la industria y en la construcción & 0,191 & 0,393 & 0,143 & 0,35 \\
\hline Operadores de instalaciones y maquinaria, y montadores & 0,083 & 0,276 & 0,098 & 0,298 \\
\hline Trabajadores no cualificados & 0,243 & 0,423 & 0,168 & 0,32 \\
\hline Actividad del establecimiento & & & & \\
\hline Agricultura, ganadería, pesca, caza,,, & 0,05 & 0,218 & 0,018 & 0,136 \\
\hline Construcción & 0,194 & 0,395 & 0,107 & 0,31 \\
\hline Industria & 0,138 & 0,257 & 0,165 & 0,343 \\
\hline Comercio y hostelería & 0,207 & 0,405 & 0,273 & 0,445 \\
\hline Transporte & 0,048 & 0,214 & 0,07 & 0,256 \\
\hline Intermediación financiera y actividades inmobiliarias & 0,097 & 0,296 & 0,143 & 0,35 \\
\hline Administraciones públicas, educación y actividades sanitarias & 0,179 & 0,383 & 0,122 & 0,327 \\
\hline Otras actividades de servicios & 0,087 & 0,283 & 0,102 & 0,302 \\
\hline Tipo de jornada & & & & \\
\hline Jornada completa & 0,787 & 0,409 & 0,846 & 0,36 \\
\hline Jornada parcial & 0,213 & 0,409 & 0,154 & 0,36 \\
\hline Comunidades Autónomas & & & & \\
\hline Andalucía & 0,237 & 0,385 & 0,139 & 0,348 \\
\hline Galicia & 0,059 & 0,236 & 0,052 & 0,223 \\
\hline Castilla-La Mancha & 0,071 & 0,258 & 0,075 & 0,264 \\
\hline Extremadura & 0,042 & 0,201 & 0,027 & 0,163 \\
\hline Valencia & 0,09 & 0,287 & 0,097 & 0,296 \\
\hline Murcia & 0,042 & 0,201 & 0,036 & 0,187 \\
\hline Canarias & 0,056 & 0,231 & 0,048 & 0,215 \\
\hline Asturias & 0,022 & 0,148 & 0,024 & 0,153 \\
\hline Cantabria & 0,023 & 0,15 & 0,024 & 0,153 \\
\hline País Vasco & 0,041 & 0,199 & 0,043 & 0,204 \\
\hline Navarra & 0,019 & 0,138 & 0,023 & 0,152 \\
\hline Aragón & 0,037 & 0,114 & 0,048 & 0,215 \\
\hline La Rioja & 0,013 & 0,19 & 0,019 & 0,138 \\
\hline Madrid & 0,052 & 0,222 & 0,085 & 0,279 \\
\hline Castilla-León & 0,077 & 0,268 & 0,088 & 0,284 \\
\hline Baleares & 0,027 & 0,164 & 0,032 & 0,176 \\
\hline Cataluña & 0,092 & 0,289 & 0,14 & 0,347 \\
\hline Año de observación & & & & \\
\hline Año 2005 & 0,205 & 0,4 & 0,177 & 0,38 \\
\hline Año 2006 & 0,22 & 0,414 & 0,186 & 0,389 \\
\hline Año 2007 & 0,221 & 0,415 & 0,216 & 0,411 \\
\hline Año 2008 & 0,201 & 0,401 & 0,225 & 0,417 \\
\hline Año 2009 & 0,153 & 0,36 & 0,196 & 0,397 \\
\hline $\mathrm{N}^{\circ}$ Observaciones & & 652 & & \\
\hline
\end{tabular}

Fuente: EPA (INE, 2005-2009). 
Tabla A2. Análisis descriptivo de asalariados españoles con menos de 3 años de antigüedad por tipo de contrato

\begin{tabular}{|c|c|c|c|c|}
\hline \multirow{2}{*}{ Variables } & \multicolumn{2}{|c|}{ Temporales } & \multicolumn{2}{|c|}{ Indefinidos } \\
\hline & Media & Desv. Est. & Media & Desv. Est. \\
\hline \multicolumn{5}{|l|}{ Sexo } \\
\hline Mujer & 0,481 & 0,5 & 0,48 & 0,5 \\
\hline Varón & 0,519 & 0,5 & 0,52 & 0,5 \\
\hline \multicolumn{5}{|l|}{ Estudios } \\
\hline Primarios o sin estudios & 0,147 & 0,32 & 0,108 & 0,29 \\
\hline Secundarios 1er Nivel y FP I & 0,355 & 0,478 & 0,31 & 0,462 \\
\hline Secundarios $2^{\circ}$ Nivel & 0,1 & 0,3 & 0,118 & 0,323 \\
\hline Grado Medio de FP & 0,101 & 0,302 & 0,113 & 0,316 \\
\hline Grado Superior de FP & 0,096 & 0,294 & 0,122 & 0,328 \\
\hline Universitarios de ciclo corto & 0,096 & 0,295 & 0,101 & 0,301 \\
\hline Universitarios de ciclo largo & 0,105 & 0,307 & 0,128 & 0,334 \\
\hline \multicolumn{5}{|l|}{ Responsabilidad familiar } \\
\hline Cabeza de familia & 0,271 & 0,444 & 0,335 & 0,472 \\
\hline No cabeza de familia & 0,729 & 0,444 & 0,665 & 0,472 \\
\hline \multicolumn{5}{|l|}{ Estado civil } \\
\hline Con pareja & 0,328 & 0,469 & 0,397 & 0,489 \\
\hline Sin pareja & 0,672 & 0,469 & 0,603 & 0,489 \\
\hline \multicolumn{5}{|l|}{ Edad potencial de actividad } \\
\hline Menos de 1 año & 0,117 & 0,3 & 0,068 & 0,25 \\
\hline Entre 1 y 3 años & 0,125 & 0,331 & 0,099 & 0,298 \\
\hline Más de 3 años & 0,758 & 0,428 & 0,833 & 0,372 \\
\hline Antigüedad en el empleo (meses) & 9,42 & 9,27 & 18,55 & 10,23 \\
\hline \multicolumn{5}{|l|}{ Ocupación } \\
\hline Directivos de las empresas y de las administraciones públicas & 0,005 & 0,075 & 0,023 & 0,152 \\
\hline Técnicos y profesionales científicos e intelectuales & 0,11 & 0,313 & 0,1 & 0,3 \\
\hline Técnicos y profesionales de apoyo & 0,103 & 0,304 & 0,147 & 0,354 \\
\hline Empleados de tipo administrativo & 0,099 & 0,298 & 0,121 & 0,326 \\
\hline Trabajadores de servicios y vendedores de comercio & 0,187 & 0,39 & 0,218 & 0,413 \\
\hline Trabajadores cualificados en la agricultura y pesca & 0,01 & 0,104 & 0,008 & 0,09 \\
\hline Trabajadores cualificados en la industria y en la construcción & 0,186 & 0,389 & 0,141 & 0,348 \\
\hline Operadores de instalaciones y maquinaria, y montadores & 0,085 & 0,279 & 0,102 & 0,279 \\
\hline Trabajadores no cualificados & 0,215 & 0,303 & 0,14 & 0,345 \\
\hline \multicolumn{5}{|l|}{ Actividad del establecimiento } \\
\hline Agricultura, ganadería, pesca, caza,,, & 0,043 & 0,203 & 0,016 & 0,128 \\
\hline Construcción & 0,181 & 0,385 & 0,105 & 0,307 \\
\hline Industria & 0,205 & 0,39 & 0,172 & 0,37 \\
\hline Comercio y hostelería & 0,273 & 0,403 & 0,272 & 0,445 \\
\hline Transporte & 0,05 & 0,219 & 0,073 & 0,261 \\
\hline Intermediación financiera y actividades inmobiliarias & 0,103 & 0,303 & 0,151 & 0,358 \\
\hline Administraciones públicas, educación y actividades sanitarias & 0,201 & 0,4 & 0,132 & 0,339 \\
\hline Otras actividades de servicios & 0,079 & 0,264 & 0,079 & 0,27 \\
\hline \multicolumn{5}{|l|}{ Tipo de jornada } \\
\hline Jornada completa & 0,781 & 0,412 & 0,846 & 0,36 \\
\hline Jornada parcial & 0,219 & 0,412 & 0,154 & 0,36 \\
\hline Comunidades Autónomas & & & & \\
\hline Andalucía & 0,166 & 0,23 & 0,147 & 0,34 \\
\hline Galicia & 0,064 & 0,224 & 0,056 & 0,23 \\
\hline Castilla-La Mancha & 0,072 & 0,258 & 0,077 & 0,267 \\
\hline Extremadura & 0,047 & 0,212 & 0,029 & 0,17 \\
\hline Valencia & 0,081 & 0,274 & 0,094 & 0,289 \\
\hline Murcia & 0,035 & 0,184 & 0,035 & 0,183 \\
\hline Canarias & 0,059 & 0,235 & 0,049 & 0,217 \\
\hline Asturias & 0,024 & 0,155 & 0,025 & 0,158 \\
\hline Cantabria & 0,023 & 0,152 & 0,024 & 0,155 \\
\hline País Vasco & 0,043 & 0,204 & 0,045 & 0,209 \\
\hline Navarra & 0,019 & 0,138 & 0,023 & 0,15 \\
\hline Aragón & 0,035 & 0,184 & 0,048 & 0,214 \\
\hline La Rioja & 0,011 & 0,107 & 0,017 & 0,131 \\
\hline Madrid & 0,044 & 0,205 & 0,077 & 0,267 \\
\hline Castilla-León & 0,079 & 0,271 & 0,091 & 0,288 \\
\hline Baleares & 0,024 & 0,155 & 0,028 & 0,165 \\
\hline Cataluña & 0,085 & 0,28 & 0,135 & 0,342 \\
\hline Año de observación & & & & \\
\hline Año 2005 & 0,212 & 0,35 & 0,185 & 0,385 \\
\hline Año 2006 & 0,221 & 0,415 & 0,189 & 0,391 \\
\hline Año 2007 & 0,219 & 0,413 & 0,215 & 0,411 \\
\hline Año 2008 & 0,197 & 0,398 & 0,22 & 0,414 \\
\hline Año 2009 & 0,151 & 0,358 & 0,191 & 0,393 \\
\hline $\mathrm{N}^{\circ}$ Observaciones & & & & \\
\hline
\end{tabular}


Tabla A3, Análisis descriptivo de asalariados de países europeos distintos a la UE15, Africa y Sudamérica con menos de 3 años de antigüedad, por tipo de contrato

\begin{tabular}{|c|c|c|c|c|}
\hline \multirow{2}{*}{ Variables } & \multicolumn{2}{|c|}{ Temporales } & \multicolumn{2}{|c|}{ Indefinidos } \\
\hline & Media & Desv. Est. & Media & Desv. Est. \\
\hline \multicolumn{5}{|l|}{ Sexo } \\
\hline Mujer & 0,431 & 0,495 & 0,584 & 0,493 \\
\hline Varón & 0,569 & 0,495 & 0,416 & 0,493 \\
\hline \multicolumn{5}{|l|}{ Estudios } \\
\hline Primarios o sin estudios & 0,253 & 0,401 & 0,193 & 0,394 \\
\hline Secundarios 1er Nivel y FP I & 0,197 & 0,397 & 0,193 & 0,394 \\
\hline Secundarios $2^{\circ}$ Nivel & 0,313 & 0,463 & 0,349 & 0,476 \\
\hline Grado Medio de FP & 0,068 & 0,251 & 0,062 & 0,241 \\
\hline Grado Superior de FP & 0,057 & 0,232 & 0,063 & 0,243 \\
\hline Universitarios de ciclo corto & 0,052 & 0,223 & 0,057 & 0,233 \\
\hline Universitarios de ciclo largo & 0,06 & 0,238 & 0,083 & 0,276 \\
\hline \multicolumn{5}{|l|}{ Responsabilidad familiar } \\
\hline Cabeza de familia & 0,416 & 0,493 & 0,404 & 0,49 \\
\hline No cabeza de familia & 0,584 & 0,493 & 0,596 & 0,49 \\
\hline \multicolumn{5}{|l|}{ Estado civil } \\
\hline Con pareja & 0,522 & 0,5 & 0,527 & 0,499 \\
\hline Sin pareja & 0,478 & 0,5 & 0,473 & 0,499 \\
\hline \multicolumn{5}{|l|}{ Edad potencial de actividad } \\
\hline Menos de 1 año & 0,205 & 0,4 & 0,138 & 0,35 \\
\hline Entre 1 y 3 años & 0,252 & 0,434 & 0,264 & 0,441 \\
\hline Más de 3 años & 0,553 & 0,497 & 0,598 & 0,49 \\
\hline Antigüedad en el empleo (meses) & 9,06 & 8,86 & & \\
\hline \multicolumn{5}{|l|}{ Ocupación } \\
\hline Directivos de las empresas y de las administraciones públicas & 0,001 & 0,03 & 0,006 & 0,08 \\
\hline Técnicos y profesionales científicos e intelectuales & 0,012 & 0,108 & 0,014 & 0,12 \\
\hline Técnicos y profesionales de apoyo & 0,017 & 0,131 & 0,031 & 0,175 \\
\hline Empleados de tipo administrativo & 0,022 & 0,148 & 0,043 & 0,204 \\
\hline Trabajadores de servicios y vendedores de comercio & 0,19 & 0,393 & 0,253 & 0,434 \\
\hline Trabajadores cualificados en la agricultura y pesca & 0,015 & 0,121 & 0,015 & 0,124 \\
\hline Trabajadores cualificados en la industria y en la construcción & 0,229 & 0,42 & 0,159 & 0,366 \\
\hline Operadores de instalaciones y maquinaria, y montadores & 0,066 & 0,249 & 0,072 & 0,259 \\
\hline Trabajadores no cualificados & 0,448 & 0,45 & 0,407 & 0,44 \\
\hline \multicolumn{5}{|l|}{ Actividad del establecimiento } \\
\hline Agricultura, ganadería, pesca, caza,,, & 0,1 & 0,3 & 0,039 & 0,193 \\
\hline Construcción & 0,282 & 0,45 & 0,126 & 0,332 \\
\hline Industria & 0,11 & 0,31 & 0,121 & 0,33 \\
\hline Comercio y hostelería & 0,219 & 0,414 & 0,274 & 0,446 \\
\hline Transporte & 0,029 & 0,17 & 0,039 & 0,195 \\
\hline Intermediación financiera y actividades inmobiliarias & 0,057 & 0,233 & 0,075 & 0,264 \\
\hline Administraciones públicas, educación y actividades sanitarias & 0,032 & 0,176 & 0,032 & 0,176 \\
\hline Otras actividades de servicios & 0,171 & 0,376 & 0,294 & 0,455 \\
\hline \multicolumn{5}{|l|}{ Tipo de jornada } \\
\hline Jornada completa & 0,822 & 0,382 & 0,843 & 0,362 \\
\hline Jornada parcial & 0,178 & 0,382 & 0,157 & 0,362 \\
\hline Comunidades Autónomas & & & & \\
\hline Andalucía & 0,108 & 0,309 & 0,06 & 0,239 \\
\hline Galicia & 0,019 & 0,138 & 0,02 & 0,141 \\
\hline Castilla-La Mancha & 0,073 & 0,261 & 0,064 & 0,245 \\
\hline Extremadura & 0,009 & 0,099 & 0,008 & 0,009 \\
\hline Valencia & 0,149 & 0,356 & 0,139 & 0,346 \\
\hline Murcia & 0,092 & 0,289 & 0,049 & 0,216 \\
\hline Canarias & 0,039 & 0,194 & 0,036 & 0,186 \\
\hline Asturias & 0,007 & 0,087 & 0,011 & 0,107 \\
\hline Cantabria & 0,019 & 0,137 & 0,014 & 0,118 \\
\hline País Vasco & 0,027 & 0,163 & 0,024 & 0,153 \\
\hline Navarra & 0,02 & 0,141 & 0,028 & 0,165 \\
\hline Aragón & 0,054 & 0,227 & 0,053 & 0,225 \\
\hline La Rioja & 0,024 & 0,155 & 0,034 & 0,183 \\
\hline Madrid & 0,107 & 0,309 & 0,147 & 0,354 \\
\hline Castilla-León & 0,065 & 0,246 & 0,073 & 0,261 \\
\hline Baleares & 0,045 & 0,209 & 0,056 & 0,23 \\
\hline Cataluña & 0,135 & 0,342 & 0,176 & 0,381 \\
\hline Año de observación & & & & \\
\hline Año 2005 & 0,159 & 0,35 & 0,121 & 0,33 \\
\hline Año 2006 & 0,211 & 0,408 & 0,161 & 0,368 \\
\hline Año 2007 & 0,235 & 0,424 & 0,221 & 0,415 \\
\hline Año 2008 & 0,228 & 0,419 & 0,263 & 0,44 \\
\hline Año 2009 & 0,167 & 0,373 & 0,234 & 0,423 \\
\hline $\mathrm{N}^{\circ}$ Observaciones & & & & 67 \\
\hline
\end{tabular}

Fuente: EPA (INE, 2005-2009). 